\title{
Learning Adaptively Context-Weight-Aware Correlation Filters for UAV Tracking with Robust Spatial-Temporal Regularization
}

\author{
Dongze Hao \\ School of Artificial Intelligence, University of Chinese \\ Academy of Sciences, Beijing, China; Institute of \\ Automation, Chinese Academy of Sciences, Beijing, China \\ Yiping Yang \\ Institute of Automation, Chinese Academy of Sciences, \\ Beijing, China
}

\begin{abstract}
Recently, Discriminative Correlation Filter (DCF) based methods have been widely applied in tracking for unmanned aerial vehicles (UAVs) because of their promising performance and efficiency. However, boundary effect, filter corruption, lack of context information and the poor representation of the object lead to the decrease in discriminability. In this paper, a novel learning adaptively contextweight-aware correlation filters with robust spatial-temporal regularization method (ACRST) is proposed. Both convolutional features and hand-crafted features are employed to improve representations for object appearances. Then the ACRST tracker extracts context samples around the object to help the filter be aware of the background information and adaptively learns the weights of these context patches. Thus, the tracker can improve the robustness against background noises especially for similar samples. Meanwhile, the tracker merges a robust spatial-temporal regularization to prevent the filter corruption and boundary effect. We design a center-attention spatial regularizer to focus on the valid information of the object better and we propose a method to obtain the value of the parameter of the temporal regularization adaptively. Extensive experiments have been conducted on 123 challenging UAV tracking sequences. The results prove that our tracker performs better than other state-of-the-art trackers.
\end{abstract}

\section{CCS CONCEPTS}

- Computing methodologies; • Artificial intelligence; • Computer vision; • Computer vision problems; • Tracking;

\section{KEYWORDS}

Correlation Filters, Adaptively Context-Weight-Aware, UAV Tracking, Robust Spatial-Temporal Regularization

* Jixiang Zhang is the Corresponding author

Permission to make digital or hard copies of all or part of this work for personal or classroom use is granted without fee provided that copies are not made or distributed for profit or commercial advantage and that copies bear this notice and the full citation on the first page. Copyrights for components of this work owned by others than ACM must be honored. Abstracting with credit is permitted. To copy otherwise, or republish, to post on servers or to redistribute to lists, requires prior specific permission and/or a fee. Request permissions from permissions@acm.org.

ICIGP 2021, January 01-03, 2021, Sanya, China

(C) 2021 Association for Computing Machinery.

ACM ISBN 978-1-4503-8910-5/21/01 ..\$15.00

https://doi.org/10.1145/3447587.3447599

\author{
Yinghao Cai \\ Institute of Automation, Chinese Academy of Sciences, \\ Beijing, China
}

Jixiang Zhang*

Institute of Automation, Chinese Academy of Sciences, Beijing, China

ACM Reference Format:

Dongze Hao, Yinghao Cai, Yiping Yang, and Jixiang Zhang. 2021. Learning Adaptively Context-Weight-Aware Correlation Filters for UAV Tracking with Robust Spatial-Temporal Regularization. In 2021 The 4th International Conference on Image and Graphics Processing (ICIGP 2021), fanuary 01-03, 2021, Sanya, China. ACM, New York, NY, USA, 9 pages. https://doi.org/10. $1145 / 3447587.3447599$

\section{INTRODUCTION}

Visual object tracking is one of the fundamental problems in computer vision. It has been widely applied in the field of unmanned aerial vehicles, such as target following [1], aerial surveillance [2], urban search and rescue [3]. The task of tracking is to estimate the position of the object in each frame given the initial bounding box of the first frame. Generally, there are many issues that influence the performance in UAV tracking, such as complex background, appearance variations caused by UAV motion, full/partial occlusion, deformation, out of view, cluttered background as well as illumination changes. Therefore, it is very necessary to design a robust tracker to overcome these issues.

Recently, the discriminative correlation filter (DCF) based tracking methods are widely adopted in UAV object tracking. The framework of DCF based method aims to train a correlation filter to discriminate the object from the background. It has high real-time performance because it uses circular shifting operation on the training and detection samples, meanwhile all the calculations are processed in the Fourier domain. Unfortunately, circular shift leads to the problem of boundary effect at the same time. To mitigate the problem of boundary effect, Danelljan et al. [4] proposed the SRDCF tracker. It introduces a spatial regularization component to penalize the values of the edge of the filter. However, it enlarges the search area and introduces the background noises. Mueller et al. [5] proposed the context-aware correlation filter tracking method(CACF). CACF tracker extracts patches around the target as the negative samples to increase the discriminative ability of the tracker. However, the weights of context patches are fixed in [5]. If similar samples appear around the target, the weights of the relevant patches should increase.

In addition, filter corruption often exists under the situation of partial occlusion and large appearance variations. Li et al. [6] proposed a temporal regularizer to restrict the filter frame by 
frame(STRCF) so that it can reduce the influence of the filter corruption. However, the value of the parameter of the temporal regularizer is fixed. If the appearance of the object varies very dramatically, the value of the parameter of the temporal regularizer should decrease to make the learned filter adapt to the current appearance. Moreover, the central coefficients of the spatial regularizer in STRCF are the same, thus STRCF cannot make the best of the valid information of the object.

In this work, a novel tracker (ACRST) is proposed to solve the aforementioned problems. The main contributions of our work are listed as follows:

- We propose a method to guide the variation of the parameter corresponding to the temporal regularizer via the response map variation, which makes the tracker more robust to situations of the dramatic appearance variations and occlusions.

- We propose a new center-attention spatial regularizer to focus on the valid information of the object and relieve the boundary effect preferably.

- We propose a new adaptively context-weight-aware learning method to obtain the weight of each context patch automatically. Thus, our tracker can better alleviate the effect of background noises.

- We evaluate our tracker on 123 challenging UAV image sequences, and the results have proven the effectiveness of our tracker.

\section{RELATED WORK}

\subsection{Discriminative correlation filter}

Recently, tracking methods based on discriminative correlation filter have achieved promising results. Meanwhile, CF-based methods have high computational efficiency. Therefore, CF-based trackers have drawn wide attention for all kinds of applications. Bolme et al. [7] firstly applied the correlation filter to the field of visual tracking, which proposed the minimum output sum of squared error (MOSSE) filter. The kernel method and the circular shifting operation were introduced to DCF framework by Henriques et al. [8], which achieves better performance. Henriques et al. [9] further incorporated the histogram of oriented gradients feature(HOG) with [8]. Then, color names $(\mathrm{CN})$ features [10] were used in visual tracking. In order to reduce the effect of the scale change and the occlusion, several methods [11] [12] [13] [14] were advanced.

\subsection{Boundary effects}

Although circular shifting operation improves the efficiency of CFbased trackers, it introduces the problem of boundary effects, which degrades the performance of CF-based trackers. Recently, several solutions are proposed to relieve this effect. Danelljan et al. [4] proposed a spatial regularization component to punish correlation filter coefficients according to the distance of their position to the center point. Galoogahi et al. [15] proposed a background-aware correlation filter(BACF). BACF learns from all possible positive and negative patches extracted from the entire frame using cropping matrix which has high computing efficiency.

\subsection{Context noise and filter corruption}

In order to relieve the influence of the background noises, Mueller et al. [5] proposed context-aware correlation filter(CACF). CACF extracts patches from surroundings of the target as negative samples to train the filter. Thus, CACF increases the discriminability and suppresses the background noises. Nevertheless, the weights of context patches are fixed and cannot change with different circumstances. Another problem of classical DCF trackers is the filter corruption. Several methods have been proposed to solve the problem, i.e.,

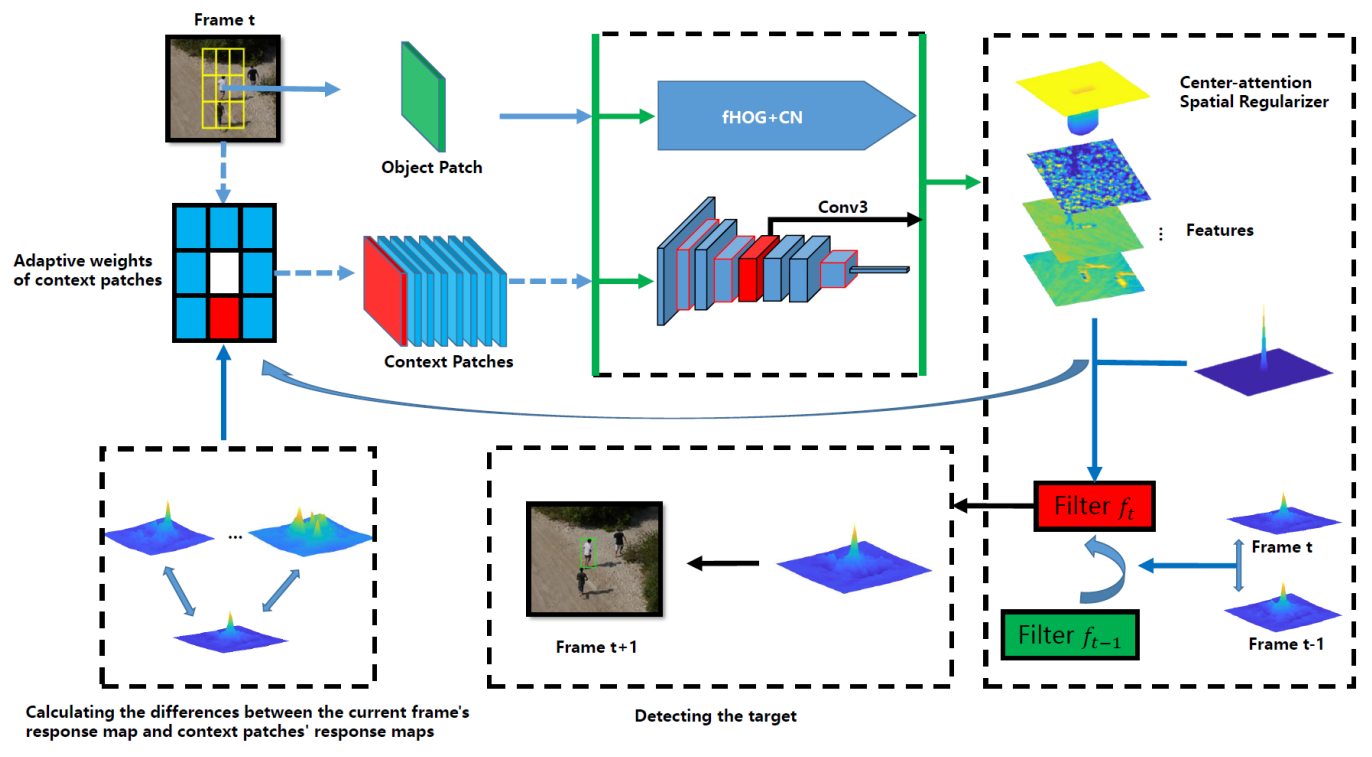

Figure 1: Main structure of our tracker. 
creating robust training set [16] [17], introducing a temporal regularization [18] [19], verifying response maps [20] [21]. Among these methods, STRCF is an effective approach. Yet the spatial regularizer in STRCF may not make the use of valid information of the object and the values of the parameters of the temporal regularizer are fixed.

\subsection{Tracking with deep neural network}

Recently, deep neural network has been widely applied in the tasks of computer vision and achieved good performance. Because of the extraordinary discriminative power of deep convolutional features, many CF-based trackers use deep convolutional features as their feature represenrations. Ma et al. [22] proposed CF2 tracker that employed features extracted from deep convolutional neural networks trained on ImageNet and improve tracking accuracy and robustness. Wang et al. [23] put forward multi-cue correlation filters. Besides, many end-to-end learning based methods are proposed. These methods directly train a neural network for visual tracking. Bertinetto et al. [24] proposed a novel fully-convolutional siamese network trained on the ILSVRC15 dataset for visual tracking. Valmadre et al. [25] interpreted the correlation filter learner to overcome the limitation that CF-based trackers adopted features which were either manually designed or trained for a different task. Although these end-to-end learning based trackers have made significant progress, they heavily depend on the training data.

\section{METHOD}

In this work, a novel adaptively context-weight-aware correlation filter for UAV tracking with robust spatial-temporal regularization is proposed. Its main structure is shown in Fig. 1 In $t$-th frame, we extract features of the target sample and context samples. Then, we obtain the reference weights of the context patches by calculating the differences between the current frame's response map and context patches' response maps. In the training process, we use the proposed center-attention spatial regularizer, adaptively context weights learning and the adaptively current filter restricting methods to get the filter. Then we use the learned filter to detect the target of the next frame.

\subsection{Overall objective of ACRST}

The overall objective of our tracker is to minimize the loss function below:

$$
\begin{gathered}
\varepsilon\left(f_{t}, \alpha_{t}\right)=\frac{1}{2}\left\|\sum_{d=1}^{D} x_{t}^{d} * f_{t}^{d}-y\right\|_{2}^{2}+\frac{1}{2} \sum_{d=1}^{D}\left\|w \cdot f_{t}^{d}\right\|_{2}^{2}+ \\
\frac{\mu}{2} \sum_{d=1}^{D}\left\|f_{t}^{d}-f_{t-1}^{d}\right\|_{2}^{2}+\frac{\alpha_{k}}{2} \sum_{k=1}^{K}\left\|\sum_{d=1}^{D} x_{k}^{d} * f_{t}^{d}\right\|_{2}^{2}+ \\
\frac{\theta}{2} \sum_{k=1}^{K}\left\|\tilde{\alpha}_{k}-\alpha_{k}\right\|_{2}^{2}
\end{gathered}
$$

where $y \in R^{M N}$ denotes the desired response map. $x_{t}^{d} \in R^{M N}$ denotes the $d$-th channel of the sample matrix from the target patch. $f_{t}^{d} \in R^{M N}$ and $f_{t-1}^{d} \in R^{M N}$ denote the $d$-th channel of the correlation filter in the $t$-th frame and $(t-1)$-th frame, respectively. $w \in R^{M N}$ denotes the spatial weight to punish the edge area of the extracted features. $x_{k}^{d} \in R^{M N}$ denotes the $d$-th channel of the sample matrix from the $k$ th context patch. $\alpha_{k}$ denotes the weight of the kth context patch. $\tilde{\alpha}_{k}$ denotes the reference weight of the corresponding context patch. $\theta$ denotes the penalty parameter of the gap between $\tilde{\alpha}_{k}$ and $\alpha_{k}$. The operator $*$ denotes the correlation operator. The operator $\cdot$ denotes the Hadamard product.

\subsection{Optimization}

In this work, we use alternating direction method of multipliers (ADMM) [33] to optimize Eqn. 1). To solve the problem, we need introduce an auxiliary variable g, requiring $f_{t}=g_{t}$. The Augmented Lagrangian form of Eqn. 1) is shown below:

$$
\begin{gathered}
\ell\left(f_{t}, \alpha_{k}, g_{t}, \zeta\right)=\frac{1}{2}\left\|\sum_{d=1}^{D} x_{t}^{d} * f_{t}^{d}-y\right\|_{2}^{2}+\frac{1}{2} \sum_{d=1}^{D}\left\|w \cdot g_{t}^{d}\right\|_{2}^{2}+ \\
\sum_{d=1}^{D}\left(f_{t}^{d}-g_{t}^{d}\right)^{T} \zeta^{d}+\frac{\gamma}{2} \sum_{d=1}^{D}\left\|f_{t}^{d}-g_{t}^{d}\right\|_{2}^{2}+\frac{\mu}{2} \sum_{d=1}^{D}\left\|f_{t}^{d}-f_{t-1}^{d}\right\|_{2}^{2}+ \\
\frac{\alpha_{k}}{2} \sum_{k=1}^{K}\left\|\sum_{d=1}^{D} x_{k}^{d} * f_{t}^{d}\right\|_{2}^{2}+\frac{\theta}{2} \sum_{k=1}^{K}\left\|\tilde{\alpha}_{k}-\alpha_{k}\right\|_{2}^{2}
\end{gathered}
$$

Where $\zeta^{d} \in R^{M N}$ is the d-th channel of the Lagrange multiplier, $\gamma$ is the penalty factor. By introducing $h=\frac{1}{\gamma} \zeta$, Eqn. 2) is equivalent to:

$$
\begin{array}{r}
\ell\left(f_{t}, \alpha_{k}, g_{t}, h\right)=\frac{1}{2}\left\|\sum_{d=1}^{D} x_{t}^{d} * f_{t}^{d}-y\right\|_{2}^{2}+\frac{1}{2} \sum_{d=1}^{D}\left\|w \cdot g_{t}^{d}\right\|_{2}^{2}+ \\
\frac{\gamma}{2} \sum_{d=1}^{D}\left\|f_{t}^{d}-g_{t}^{d}+h_{t}^{d}\right\|_{2}^{2}+\frac{\mu}{2} \sum_{d=1}^{D}\left\|f_{t}^{d}-f_{t-1}^{d}\right\|_{2}^{2}+ \\
\frac{\alpha_{k}}{2} \sum_{k=1}^{K}\left\|\sum_{d=1}^{D} x_{k}^{d} * f_{t}^{d}\right\|_{2}^{2}+\frac{\theta}{2} \sum_{k=1}^{K}\left\|\tilde{\alpha}_{k}-\alpha_{k}\right\|_{2}^{2}
\end{array}
$$

Then, the ADMM algorithm is adopted by alternately solving the following subproblems:

Subproblem $\mathbf{f}$ : the optimal $f_{t}^{*}$ can be obtained as:

$$
\begin{array}{r}
f_{t}^{*}=\arg \min _{f_{t}} \frac{1}{2}\left\|\sum_{d=1}^{D} x_{t}^{d} * f_{t}^{d}-y\right\|_{2}^{2}+\frac{\gamma}{2} \sum_{d=1}^{D}\left\|f_{t}^{d}-g_{t}^{d}+h_{t}^{d}\right\|_{2}^{2}+ \\
\frac{\mu}{2} \sum_{d=1}^{D}\left\|f_{t}^{d}-f_{t-1}^{d}\right\|_{2}^{2}+\frac{\alpha_{k}}{2} \sum_{k=1}^{K}\left\|\sum_{d=1}^{D} x_{k}^{d} * f_{t}^{d}\right\|_{2}^{2}
\end{array}
$$

By applying Parsevals theorem, Eqn. 4) can be transformed into Fourier domain as:

$$
\begin{array}{r}
\widehat{f}_{t}^{*}=\arg \underset{\widehat{f}_{t}}{\min } \frac{1}{2}\left\|\sum_{d=1}^{D} \hat{x}_{t}^{d} \cdot \widehat{\widetilde{f}}_{t}^{d}-\widehat{y}\right\|_{2}^{2}+\frac{\gamma}{2} \sum_{d=1}^{D}\left\|\widehat{f}_{t}^{d}-\widehat{g}_{t}^{d}+\widehat{h}_{t}^{d}\right\|_{2}^{2}+ \\
\frac{\mu}{2} \sum_{d=1}^{D}\left\|\widetilde{f}_{t}^{d}-\widehat{f}_{t-1}^{d}\right\|_{2}^{2}+\frac{\alpha_{k}}{2} \sum_{k=1}^{K}\left\|\sum_{d=1}^{D} \widehat{x}_{k}^{d} \cdot \widehat{f}_{t}^{d}\right\|_{2}^{2}
\end{array}
$$

where the hat ${ }^{\wedge}$ denotes the discrete Fourier transform(DFT) operation. It will be time-consuming to directly optimize Eqn. 5). On account of the $j$-th element of $\hat{y}$ only relies solely on the $j$-th element of $\hat{f}_{t}$ and $\hat{x}_{t}$ across all D channels, we can divide Eqn. 5) into MN independent subproblems as :

$$
\begin{gathered}
V_{j}^{*}\left(\widehat{f}_{t}\right)=\arg \min _{V_{j}\left(\widehat{f}_{t}\right)} \frac{1}{2}\left\|V_{j}\left(\widehat{x}_{t}\right)^{H} V_{j}\left(\widehat{f}_{t}\right)-\widehat{y}_{j}\right\|_{2}^{2}+\frac{\gamma}{2} \| V_{j}\left(\hat{f}_{t}\right)-V_{j}\left(\widehat{g}_{t}\right)+ \\
V_{j}\left(\widehat{h}_{t}\right)\left\|_{2}^{2}+\frac{\mu}{2}\right\| V_{j}\left(\hat{f}_{t}\right)-V_{j}\left(\hat{f}_{t-1}\right)\left\|_{2}^{2}+\frac{\alpha_{k}}{2} \sum_{k=1}^{K}\right\| V_{j}\left(\widehat{x}_{k}\right)^{H} V_{j}\left(\widehat{f}_{t}\right) \|_{2}^{2}
\end{gathered}
$$


where $V_{j}^{*}\left(\hat{f}_{t}\right) \epsilon R^{D}$ denotes the vector consisting of the $j$-th elements of $\hat{f}_{t}$ across all $\mathrm{D}$ channels. The operation $\mathrm{H}$ denotes Hermitian transpose. Then, the solution of each subproblem can be obtained as:

$$
V_{j}^{*}\left(\widehat{f}_{t}\right)=\left(\sum_{k=0}^{K} \alpha_{k} V_{j}\left(\widehat{x}_{k}\right) V_{j}\left(\widehat{x}_{k}\right)^{H}+(\mu+\gamma) I\right)^{-1} q,
$$

where $x_{0}$ equals to $x_{t}, \alpha_{0}=1 . q=V_{j}\left(\widehat{x}_{t}\right) \widehat{y}_{j}+\gamma V_{j}\left(\widehat{g}_{t}\right)-\gamma V_{j}\left(\widehat{h}_{t}\right)+$ $\mu V_{j}\left(\widehat{f}_{t-1}\right)$. Eqn. 6) can be solved with the Woodbury formula to improve the computational efficiency:

$$
V_{j}^{*}\left(\tilde{f}_{t}\right)=\frac{1}{\mu+\gamma}\left(I-\frac{\sum_{k=0}^{K} \alpha_{k} V_{j}\left(\widehat{x}_{k}\right) V_{j}\left(\widehat{x}_{k}\right)^{H}}{\mu+\gamma+\sum_{k=0}^{K} \alpha_{k} V_{j}\left(\widehat{x}_{k}\right) V_{j}\left(\widehat{x}_{k}\right)^{H}}\right) q .
$$

Subproblem g: $g_{t}^{*}$ can be solved in spatial domain. By setting partial derivative to zero, we can obtain:

$$
\begin{gathered}
g_{t}^{*}=\arg \min _{g_{t}} \frac{1}{2} \sum_{d=1}^{D}\left\|w \cdot g_{t}^{d}\right\|_{2}^{2}+\frac{\gamma}{2} \sum_{d=1}^{D}\left\|f_{t}^{d}-g_{t}^{d}+h_{t}^{d}\right\|_{2}^{2} \\
=\frac{\gamma\left(f_{t}+h_{t}\right)}{w^{T} w+\gamma} .
\end{gathered}
$$

where $\mathrm{w}$ denotes a $\mathrm{DMN} \times \mathrm{DMN}$ matrix.

Subproblem $\alpha_{k}$ : given $\tilde{\alpha}_{k}, f_{t}^{d}, x_{k}^{d}$, the optimal solution of $\alpha_{k}$ can be determined as:

$$
\begin{gathered}
\alpha_{k}^{*}=\arg \min _{\alpha_{k}} \frac{\alpha_{k}}{2}\left\|\sum_{d=1}^{D} x_{k}^{d} * f_{t}^{d}\right\|_{2}^{2}+\frac{\theta}{2}\left\|\tilde{\alpha}_{k}-\alpha_{k}\right\|_{2}^{2} \\
=\tilde{\alpha}_{k}-\frac{1}{2 \theta}\left\|\sum_{d=1}^{D} x_{k}^{d} * f_{t}^{d}\right\|_{2}^{2} .
\end{gathered}
$$

Lagrangian Multiplier Update: we update Lagrangian Multiplier as:

$$
\widehat{\hat{h}}^{(i+1)}=\widehat{\hat{h}}^{(i)}+\gamma^{(i)}\left(\widehat{f}^{(i+1)}-\widehat{g}^{(i+1)}\right) .
$$

where $\mathrm{i}$ and $\mathrm{i}+1$ denote the ADMM iteration index and the step parameter $\gamma$ is updated as:

$$
\gamma^{(i+1)}=\min \left(\gamma^{\max }, \beta \gamma^{(i)}\right) .
$$

where $\gamma^{\max }$ is the maximum value of $\gamma$ and $\beta$ denotes a scale factor.

Thus, after applying these four steps, we can obtain the current filter $\hat{f}_{t}$. Then we can use the filter to detect the target and store $\hat{f}_{t}$ as $\hat{f}_{\text {pre }}$ of the next frame.

\subsection{Selection of context patches and reference weighting scheme}

We select eight context patches around the object. To improve the efficiency of the algorithm, we train the filter with adaptively context-weight-aware learning method every eight frames.

Before using ADMM algorithm to train the model, we give a reference weight for each context patch. We use the differences between the response maps of the target patch and the context patch to determine the value of the context weight. In the first frame, we will initialize the weights of the context patches with the same value $\alpha_{\text {initial }}$ and the weights are automatically changed in the training process. Then every eight frames, we firstly initialize the weights of the context patches with the same value as the first frame. Next, the correlation filter learned in the previous frame is used to filter with the detected target to produce a response map. Meanwhile, the filter also filters with each context patch to generate the corresponding response map. The difference is calculated by following formula:

$$
\text { Diff } f_{k}=e^{-\left\|M_{t}-M_{k}\right\|_{2}^{2}} .
$$

where $M_{t}$ denotes the response map of the target and $M_{k}$ denotes the response map of the $k$-th context patch. Through Eqn. 13), we can calculate the response map difference of each context patch. Then we will give the reference weight according to these differences:

$$
\tilde{\alpha}_{k}=\left\{\begin{array}{cc}
\alpha_{\text {relative }} & \text { ifDif } f_{k}>=\phi \\
\alpha_{\text {initial }} & \text { others }
\end{array}\right.
$$

If $\operatorname{Dif} f_{k}$ is greater than the threshold $\emptyset$, it means that the response map of $k$-th context patch is similar to the response map of the object. Therefore, we need to give a relatively high weight as the reference weight.

\subsection{Robust spatial-temporal regularization setup}

Center-attention spatial regularization: In STRCF, the spatial regularizer $w_{0}$ has limitation, which is defined as:

$$
w^{0}(x, y)=\left\{\begin{array}{lc}
w_{\min }^{0} & \text { if }(x, y)<\Omega \\
w_{\max }^{0} & \text { others }
\end{array}\right.
$$

where $\Omega$ denotes the region of bounding box and $w_{\text {max }}^{0} \gg w_{\text {min }}^{0}$. Thus, the correlation filter only focuses on the region of bounding box. Yet, there are still invalid information at the edge of the bounding box. Coefficients of $w_{0}$ in the bounding box are the same as shown in Fig. 2(b). To focus on the central valid information of the object, a center-attention spatial regularizer is proposed as follows:

$$
w^{1}(x, y)=\left\{\begin{array}{cc}
w_{\min }^{1}+\eta\left(\frac{x^{2}}{m^{2}}+\frac{y^{2}}{n^{2}}\right), & \text { if }(x, y)<\Omega \\
w_{\max }^{1} . & \text { others }
\end{array}\right.
$$

where $\Omega$ is the same as above, $\mathrm{m}$ and $\mathrm{n}$ denote the width and height of the sample patch, $w_{\text {max }}^{1} \gg w_{\text {min }}^{1}$.

As Fig. 2(b) shows, our spatial regularizer pays more attention to the central area and suppresses futile boundary information. The ablation study has proven the effectiveness of our improvement.

Adaptively temporal regularization: In STRCF, the parameters of the temporal regularization are fixed. However, if the appearance of the object changes very drastically, the correlation filter should learn more rapidly. Furthermore, if occlusion occurs, the correlation filter should stop to learn. Therefore, the value of the parameter of the temporal regularization should change adaptively under different situations. We use the response map variations of the current frame and the last frame to adjust the value. The difference of two response maps $R_{1}$ and $R_{2}$ is calculated as follows:

$$
D=e^{-|| R_{1}[\psi \Delta]-R_{2} \|_{2}^{2}} .
$$

where $[\psi \Delta]$ indicates the shifting operation in order to make the two peaks of the two response maps coincide with each other. When the similarity of two response maps drops, the value of Eqn. 17) will be high. According to the value of Eqn. 17), we can define an 


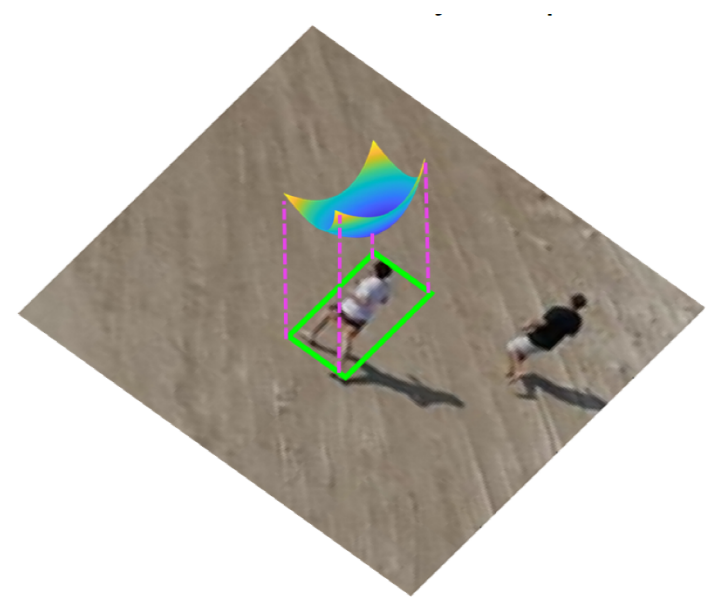

(a)

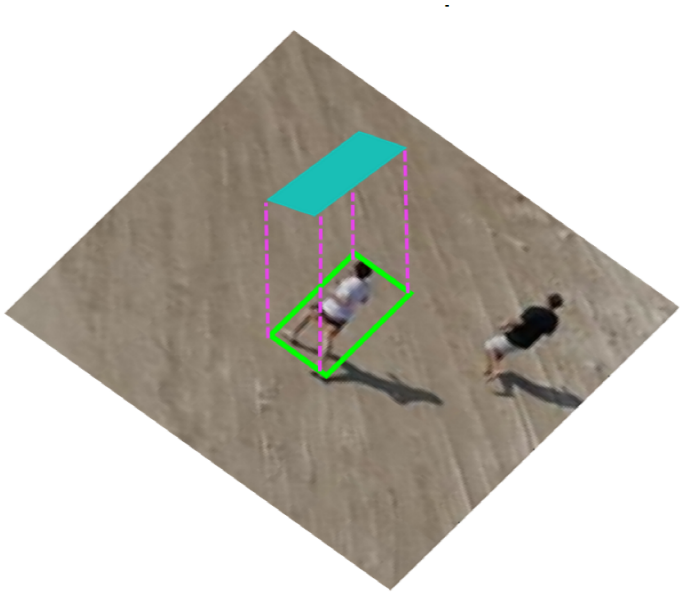

(b)

Figure 2: Visualization of spatial regularization w1 and w0 in bounding box region.

adaptive parameter of the temporal regularization $\mu$ :

$$
\mu=\left\{\begin{array}{cc}
\frac{\mu_{\text {initial }}}{1+\log (p(1-D)+1)} & \text { if } D>=\Phi \\
\mu_{\max } & \text { others }
\end{array}\right.
$$

where $\mu_{\text {initial }}$ is the given parameter of the temporal regularization in the beginning, $\mu$ denotes the adjusted parameter. $\mathrm{p}$ is defined as a zoom factor to make the value of the parameter varies within a proper range. If $\mathrm{D}$ is lower than the threshold $\Phi$, it means that there are occlusions or other aberrances, the value should be very large to prevent correlation filter to learn. If D is higher than the threshold $\Phi$, the higher $D$ is, the more dramatic appearance of the object varies, the value of $\mu$ should be small to make correlation filter learn more rapidly.

\section{EXPERIMENTS}

In this section, we evaluate the tracking performance of the proposed ACRST tracker on 123 challenging UAV image sequences from UAV123@10fps [26]. Experimental results are compared to 20 state-of-the-art trackers based on handcrafted features or deep learning, i.e., KCF [9], DCF [9], KCC [27], fDSST [11], SAMF [12], STAPLE [28], STAPLE-CA [5], MCCT-H [23], MCCT [23], SRDCF [4], BACF [15], ECO-HC [16], STRCF [6], DeepSTRCF [6], ASRCF [29], UDT [30], UDT+ [30], SiameseFC [24], CFNet [25], CF2 [22].

\subsection{Evaluation criteria}

To evaluate the performance of a tracker, Center location error(CLE) and success rate for one-pass evaluation (OPE) are employed [31]. The CLE is obtained via the Euclidian distance between the center points of estimated bounding boxes and manually annotated groundtruth. The success rate is calculated via the intersection over union (IoU) of estimated bounding boxes and manually annotated groundtruth. The precision plot (PP) and the success plot (SP) are used to illustrate the results vividly. PP shows the percentage of frames whose estimated location is within the given threshold distance of the ground truth. In this work we use the score for the threshold $=20$ pixels. SP is the ratios of successful frames whose IoU is higher than a certain value that varied from 0 to 1 and this work uses the area under curve (AUC) of each success plot to rank all trackers. In this paper, we use DS and SS to represent the distance precision score and success rate score for simplicity.

\subsection{Implementation details}

All the experiments are implemented by MATLAB R2018b on the computer with an $\mathrm{i} 7-5820 \mathrm{~K}(3.30 \mathrm{KHz}) \mathrm{CPU}, 32 \mathrm{~GB}$ RAM and a single TITAN X GPU. The MatConvNet toolbox [32] is used for extracting the deep convolutional features. For the hyper-parameters in the experiment, they are shown in Table 1

\subsection{Overall performance evaluation}

4.3.1 Quantitative and qualitative evaluation. Fig. 3 demonstrates the precision plots and success plots of our tracker. Quantitative results of overall and attribute-based DS and SS are shown in Table. 2 and Table. 3. In both DS and SS, ACRST tracker ranks first among all the trackers. As shown in the precision plots(Fig.2(a)), ACRST(0.716) tracker outperforms the second tracker MCCT(0.687) and the third tracker ASRCF(0.686) by $4.2 \%$ and $4.4 \%$. As shown in the success plots(Fig.2(b)), ACRST(0.512) outperforms the second tracker DeepSTRCF $(0.499)$ and the third tracker MCCT(0.495) by $2.6 \%$ and $3.4 \%$. In a word, with powerful deep convolutional feature, adaptively context-weight-aware learning method and robust spatial-temporal regularization, ACRST exhibits a competitive performance with other trackers. Fig. 4 shows the qualitative results of ACRST and some other top trackers.

4.3.2 Attribute-based evaluation. All the sequences are labeled with aspect ratio change (ARC), background clutter (BC), scale variation (SV), similar object (SOB), illumination variation (IV), full occlusion (FOC), partial occlusion (POC), camera motion (CM), fast motion (FM), low resolution (LR), out-of-view (OV), viewpoint 
Table 1: The value of main parameters in ACRST.

\begin{tabular}{llllll}
\hline Parameter & Value & Parameter & Value & Parameter & Value \\
\hline$\mu_{\text {initial }}$ & 17 & $\mu_{\text {max }}$ & 50 & $\theta$ & -0.5 \\
$\alpha_{\text {initial }}$ & 0.28 & $\alpha_{\text {relative }}$ & 0.5 & ADMM iterations & 2 \\
$\gamma^{0}$ & 1 & $\gamma^{\max }$ & 10 & $\beta$ & 0.1 \\
Scale step & 1.01 & Number of scales & 5 & threshold $\phi$ & 0.5 \\
threshold $\Phi$ & 0.5 & $\mathrm{P}$ & 1 & & \\
\hline
\end{tabular}

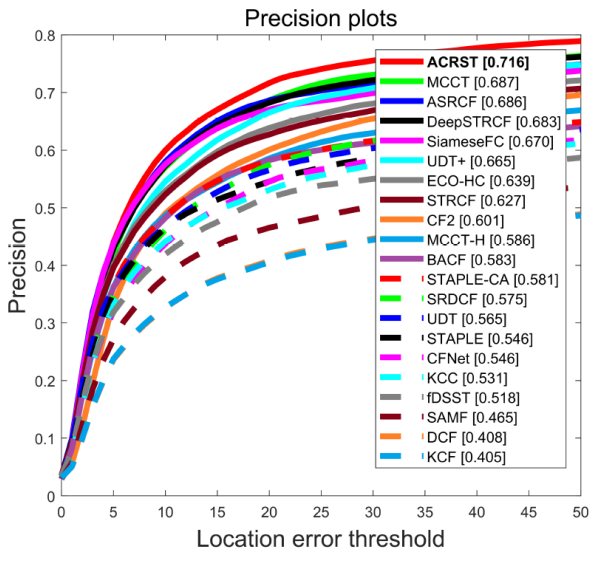

(a)

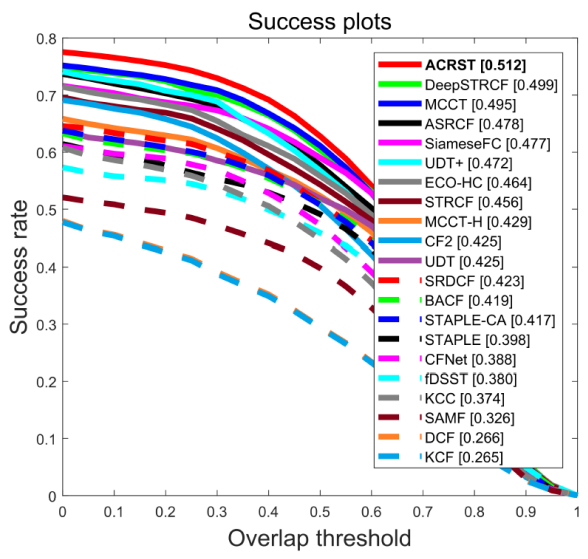

(b)

Figure 3: Precision and success plots of ACRST and other state-of-the-art trackers.
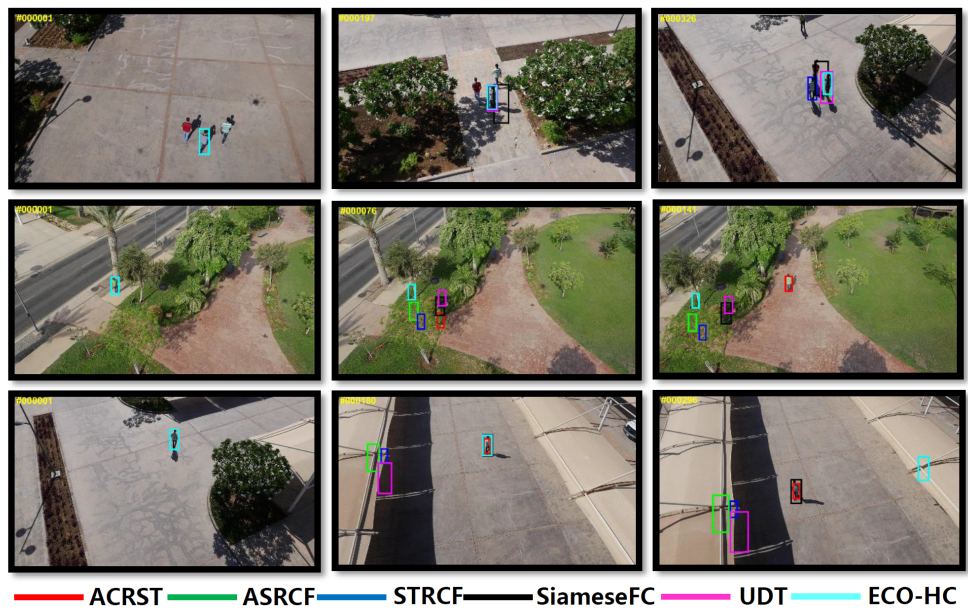

Figure 4: Qualitative evaluation on group1_1, person16, person12_2. UAV tracking video is https://youtu.be/56QXVNbMUKM.

change (VC). The attribute based performance of ACRST and other state-of-the-art trackers is evaluated.

Table 2 shows the DS of 12 attributes. ACRST ranks first among the 10 attributes out of 12 , including $\operatorname{ARC}(0.659), \mathrm{CM}(0.711)$, $\mathrm{FM}(0.626), \mathrm{FOC}(0.519), \mathrm{LR}(0.567), \mathrm{OV}(0.642), \mathrm{POC}(0.661), \mathrm{SV}(0.680)$, $\mathrm{SOB}(0.734)$, VC(0.673). With multiple experts, MCCT performs best for BC and IV. Table 3 shows the SS of 12 attributes. ACRST ranks first among the 9 attributes out of 12 , including $\mathrm{ARC}(0.452)$, $\mathrm{CM}(0.518), \mathrm{FM}(0.417), \mathrm{LR}(0.322), \mathrm{OV}(0.642), \mathrm{POC}(0.448), \mathrm{SV}(0.479)$, $\mathrm{SOB}(0.511), \mathrm{VC}(0.482)$. In sequences with BC, FOC and IV, MCCT ranks first among all the trackers.

In the situation of SOB, ACRST has shown a great improvement from baseline STRCF and has the greatest performance of all the 
Table 2: DS of ACRST and other state-of-the-art trackers. Red, Green, Blue respectively represent the first, second and third place. The speed is measured by fps. DL presents deep learning and CF presents correlation filter.

\begin{tabular}{|c|c|c|c|c|c|c|c|c|c|c|c|c|c|c|c|c|}
\hline Tracker & Speed & DL & $\mathrm{CF}$ & ARC & $\mathrm{BC}$ & $\mathrm{CM}$ & FM & FOC & IV & LR & $\mathrm{OV}$ & POC & SV & SOB & $\mathrm{VC}$ & Overall \\
\hline MCCT & 7.2 & $\sqrt{ }$ & $\sqrt{ }$ & 60.6 & 56.0 & 67.8 & 55.8 & 51.4 & 65.4 & 52.2 & 59.5 & 64.0 & 64.6 & 70.5 & 63.0 & 68.7 \\
\hline ASRCF & 19.9 & $\sqrt{ }$ & $\sqrt{ }$ & 60.7 & 54.7 & 66.2 & 56.6 & 64.4 & 61.5 & 56.5 & 54.3 & 60.2 & 64.6 & 70.1 & 62.2 & 68.6 \\
\hline DeepSTRCF & 4.8 & $\sqrt{ }$ & $\sqrt{ }$ & 61.0 & 53.8 & 67.2 & 59.4 & 49.9 & 59.3 & 54.2 & 61.2 & 62.5 & 64.2 & 69.5 & 60.5 & 68.3 \\
\hline SiameseFC & 12.0 & $\sqrt{ }$ & & 60.8 & 43.5 & 62.9 & 57.2 & 47.4 & 61.4 & 50.9 & 62.4 & 58.0 & 63.7 & 62.1 & 62.0 & 67.0 \\
\hline UDT+ & 43.0 & $\sqrt{ }$ & & 59.3 & 55.9 & 61.7 & 47.2 & 49.3 & 58.9 & 55.4 & 53.6 & 60.1 & 62.3 & 67.2 & 56.9 & 66.5 \\
\hline ECO_HC & 54.4 & & $\sqrt{ }$ & 56.8 & 54.6 & 61.6 & 47.5 & 47.8 & 51.4 & 52.9 & 52.3 & 56.9 & 59.2 & 65.6 & 55.7 & 63.9 \\
\hline STRCF & 20.9 & & $\sqrt{ }$ & 52.2 & 47.8 & 60.0 & 48.0 & 43.0 & 49.1 & 50.7 & 52.5 & 56.0 & 58.0 & 63.2 & 53.6 & 62.7 \\
\hline CF2 & 1.7 & $\sqrt{ }$ & $\sqrt{ }$ & 50.5 & 49.0 & 57.5 & 40.8 & 48.4 & 55.0 & 43.9 & 50.6 & 54.6 & 55.0 & 62.8 & 54.2 & 60.1 \\
\hline MCCT_H & 39.2 & & $\sqrt{ }$ & 60.6 & 45.2 & 52.5 & 36.2 & 39.2 & 46.0 & 44.8 & 46.4 & 53.5 & 54.2 & 60.8 & 46.7 & 58.6 \\
\hline $\mathrm{BACF}$ & 25.6 & & $\sqrt{ }$ & 48.9 & 42.6 & 54.0 & 40.3 & 34.8 & 42.5 & 43.9 & 44.1 & 48.3 & 53.7 & 64.0 & 49.4 & 58.3 \\
\hline STAPLE_CA & 43.3 & & $\sqrt{ }$ & 47.0 & 42.1 & 50.1 & 32.1 & 38.1 & 44.3 & 43.8 & 44.0 & 50.6 & 52.8 & 62.6 & 49.2 & 58.1 \\
\hline SRDCF & 3.2 & & $\sqrt{ }$ & 47.2 & 38.9 & 52.7 & 42.7 & 41.8 & 43.6 & 43.1 & 49.2 & 50.4 & 53.1 & 58.5 & 47.4 & 57.5 \\
\hline UDT & 44.8 & $\sqrt{ }$ & & 47.8 & 34.9 & 50.9 & 42.3 & 39.9 & 38.8 & 47.5 & 46.6 & 48.7 & 53.1 & 60.3 & 43.3 & 56.5 \\
\hline STAPLE & 67.4 & & $\sqrt{ }$ & 42.1 & 40.9 & 46.5 & 28.0 & 36.7 & 39.7 & 39.1 & 44.0 & 48.6 & 48.8 & 55.7 & 45.3 & 54.6 \\
\hline CFNet & 17.7 & $\sqrt{ }$ & & 45.2 & 36.7 & 47.6 & 37.5 & 37.6 & 44.4 & 39.0 & 43.5 & 46.9 & 49.6 & 59.2 & 46.3 & 54.6 \\
\hline KCC & 55.5 & & $\sqrt{ }$ & 43.1 & 36.2 & 46.0 & 34.2 & 35.2 & 40.7 & 40.1 & 37.3 & 45.5 & 47.9 & 54.1 & 45.1 & 53.1 \\
\hline fDSST & 81.6 & & $\sqrt{ }$ & 42.2 & 32.2 & 43.4 & 35.1 & 38.0 & 39.7 & 40.0 & 44.9 & 45.2 & 47.3 & 53.5 & 40.4 & 51.8 \\
\hline SAMF & 3.9 & & $\sqrt{ }$ & 39.0 & 27.4 & 37.8 & 33.2 & 37.9 & 33.9 & 32.2 & 40.9 & 41.9 & 44.1 & 50.3 & 35.8 & 46.5 \\
\hline DCF & 166.6 & & $\sqrt{ }$ & 30.9 & 22.6 & 30.7 & 23.0 & 28.3 & 27.4 & 30.2 & 30.9 & 34.6 & 37.5 & 45.8 & 32.0 & 40.8 \\
\hline $\mathrm{KCF}$ & 121.3 & & $\sqrt{ }$ & 30.3 & 22.3 & 30.5 & 21.7 & 28.1 & 26.9 & 30.5 & 30.9 & 34.4 & 37.3 & 45.3 & 31.6 & 40.5 \\
\hline ACRST & 4.4 & $\sqrt{ }$ & $\sqrt{ }$ & 65.9 & 53.5 & 71.1 & 62.6 & 51.9 & 64.2 & 56.7 & 64.2 & 66.1 & 68.0 & 73.4 & 67.3 & 71.6 \\
\hline
\end{tabular}

trackers. This is because adaptively context-weight-aware learning method suppresses background distraction especially similar objects to improve the discriminant ability of the tracker. In the situations of POC and OV, ACRST has the best DS and SS. And in FOC, our tracker has the first DS and the second SS that is almost equal to top one. This shows that with our robust spatial-temporal regularization, our tracker performs much better than baseline STRCF in the situation of large appearance variation.

\subsection{Ablation study and key parameter analysis}

4.4.1 Ablation study. In order to verify the effectiveness of the different modules in our proposed ACRST tracker, the ablation study is conducted. Table 4 shows the DS and SS of the ACRST with different components. RT denotes the proposed method that adjusts the value of parameter of the temporal regularization adaptively. RS denotes the proposed center-attention spatial regularization. ACL denotes the proposed adaptively context-weight-aware learning method. DF denotes deep convolution features.

As shown in Table. 4, ACRST_basic is the ACRST tracker with none of the four modules and has just moderate performance. Based on ACRST_basic, RT module is added to the ACRST_basic, ACRST_RT has an improvement in both DS and SS. The proposed RT method results in a DS enhancement of $1.4 \%$ and a SS enhancement of $1.8 \%$. Then the RS module is enforced, DS and SS have increased by $1.7 \%$ and $0.9 \%$ respectively. Next, ACRST_RT\&RS tracker with ACL module has an improvement in both DS of $0.9 \%$ and SS of $1.3 \%$. At the last step, with powerful deep convolution features, ACRST_full has further enhanced the tracking performance. ACRST_full outperforms ACRST_RT\&RS\&ACL by $9.6 \%$ in DS and
$8 \%$ in SS. ACRST achieves the best performance compared with other state-of-the-art trackers.

4.4.2 Key parameter analysis. As shown in Fig. 5, the value of $\mu_{\text {initial }}$ in Eq. 18 has a great influence on the DS and SS of the tracker. $\mu_{\text {initial }}$ affects the range of the adjusted parameter $\mu$. When $\mu_{\text {initial }}$ is too small, the final $\mu$ would be very small that causes correlation filter learning very rapidly. Thus, the trained correlation filter will degenerate. When $\mu_{\text {initial }}$ is too large, the learned correlation filter will be restricted to the previous filter greatly, which make the tracker cannot handle the situation of large appearance variation robustly. Therefore, a proper value of $\mu_{\text {initial }}$ is necessary. In our analysis, $\mu_{\text {initial }}$ ranges from 10 to 21 with a step size of 1 . Our tracker has the best DS and SS if $\mu_{\text {initial }}$ equals to 17 .

\section{CONCLUSION}

In this work, we propose an adaptively context-weight-aware correlation filters with robust spatial-temporal regularization method for UAV tracking. By designing a center-attention spatial regularizer, our tracker can focus on the important information of the object and mitigate the boundary effect better. By presenting a method adjusting the value of the parameter of the temporal regularization adaptively, our tracker can robustly adapt to different situations like large appearance variation, occlusion and so on. By introducing the adaptively context-weight-aware learning method, our tracker has a more robust discriminant ability to distinguish the target from the background. Extensive experiments on 123 sequences have proven 
Table 3: SS of ACRST and other state-of-the-art trackers. Red, Green, Blue respectively represent the first, second and third place. The speed is measured by fps. DL presents deep learning and CF presents correlation filter.

\begin{tabular}{|c|c|c|c|c|c|c|c|c|c|c|c|c|c|c|c|c|}
\hline Tracker & Speed & DL & $\mathrm{CF}$ & ARC & $\mathrm{BC}$ & $\mathrm{CM}$ & FM & FOC & IV & LR & $\mathrm{OV}$ & POC & SV & SOB & $\mathrm{VC}$ & Overall \\
\hline MCCT & 7.2 & $\sqrt{ }$ & $\sqrt{ }$ & 42.2 & 37.8 & 49.4 & 37.9 & 29.5 & 44.7 & 30.6 & 44.1 & 44.6 & 46.1 & 49.7 & 45.3 & 49.5 \\
\hline ASRCF & 19.9 & $\sqrt{ }$ & $\sqrt{ }$ & 40.2 & 33.8 & 47.4 & 36.5 & 24.9 & 40.6 & 31.4 & 39.0 & 40.7 & 44.3 & 48.5 & 42.1 & 47.8 \\
\hline DeepSTRCF & 4.8 & $\sqrt{ }$ & $\sqrt{ }$ & 43.5 & 35.4 & 50.0 & 40.1 & 28.0 & 41.7 & 31.5 & 45.1 & 43.2 & 46.5 & 49.7 & 45.1 & 49.9 \\
\hline SiameseFC & 12.0 & $\sqrt{ }$ & & 41.0 & 28.5 & 45.6 & 37.7 & 25.3 & 39.8 & 31.1 & 43.7 & 39.3 & 44.8 & 43.2 & 44.2 & 47.7 \\
\hline UDT+ & 43.0 & $\sqrt{ }$ & & 41.2 & 35.2 & 44.9 & 32.3 & 28.1 & 39.6 & 31.1 & 40.6 & 41.7 & 44.0 & 47.2 & 40.2 & 47.2 \\
\hline ECO_HC & 54.4 & & $\sqrt{ }$ & 39.8 & 36.2 & 45.2 & 31.8 & 26.5 & 36.7 & 30.0 & 38.9 & 40.0 & 42.7 & 47.9 & 40.2 & 46.4 \\
\hline STRCF & 20.9 & & $\sqrt{ }$ & 37.1 & 31.8 & 44.2 & 32.5 & 23.4 & 35.3 & 29.0 & 38.8 & 38.8 & 41.8 & 45.6 & 39.3 & 45.6 \\
\hline CF2 & 1.7 & $\sqrt{ }$ & $\sqrt{ }$ & 34.7 & 30.2 & 41.6 & 28.4 & 26.0 & 38.0 & 22.9 & 37.4 & 36.8 & 38.7 & 43.6 & 38.1 & 42.5 \\
\hline MCCT_H & 39.2 & & $\sqrt{ }$ & 36.2 & 29.6 & 39.9 & 26.3 & 22.0 & 33.4 & 25.7 & 35.3 & 37.5 & 39.6 & 44.3 & 35.0 & 42.9 \\
\hline $\mathrm{BACF}$ & 25.6 & & $\sqrt{ }$ & 33.8 & 27.6 & 39.9 & 27.0 & 17.6 & 30.5 & 25.0 & 32.9 & 33.7 & 38.1 & 44.3 & 35.3 & 41.9 \\
\hline STAPLE_CA & 43.3 & & $\sqrt{ }$ & 33.4 & 28.2 & 37.7 & 21.9 & 20.3 & 32.1 & 23.7 & 34.3 & 34.7 & 37.3 & 44.6 & 35.8 & 41.7 \\
\hline SRDCF & 3.2 & & $\sqrt{ }$ & 34.6 & 26.3 & 39.9 & 31.1 & 22.9 & 33.3 & 23.7 & 36.8 & 35.5 & 39.0 & 42.1 & 35.6 & 42.3 \\
\hline UDT & 44.8 & $\sqrt{ }$ & & 35.7 & 23.4 & 38.4 & 29.0 & 21.9 & 29.0 & 29.9 & 35.0 & 34.9 & 39.9 & 43.4 & 33.9 & 42.5 \\
\hline STAPLE & 67.4 & & $\sqrt{ }$ & 31.1 & 27.4 & 35.3 & 19.3 & 19.9 & 30.4 & 21.4 & 32.7 & 33.7 & 35.4 & 41.3 & 34.0 & 39.8 \\
\hline CFNet & 17.7 & $\sqrt{ }$ & & 31.8 & 22.3 & 34.6 & 24.5 & 20.0 & 30.0 & 21.3 & 32.2 & 31.9 & 35.2 & 41.0 & 33.3 & 38.8 \\
\hline KCC & 55.5 & & $\sqrt{ }$ & 30.2 & 23.7 & 33.0 & 21.7 & 18.5 & 30.3 & 21.3 & 37.3 & 30.2 & 33.1 & 37.5 & 32.5 & 37.4 \\
\hline fDSST & 81.6 & & $\sqrt{ }$ & 30.4 & 21.0 & 32.5 & 23.9 & 19.7 & 29.5 & 22.7 & 31.7 & 31.3 & 34.5 & 38.7 & 30.4 & 38.0 \\
\hline SAMF & 3.9 & & $\sqrt{ }$ & 27.4 & 16.0 & 27.1 & 22.0 & 19.7 & 23.8 & 16.6 & 28.9 & 28.2 & 30.6 & 34.8 & 26.4 & 32.6 \\
\hline DCF & 166.6 & & $\sqrt{ }$ & 20.3 & 12.6 & 21.1 & 15.4 & 13.5 & 18.5 & 14.6 & 22.2 & 22.6 & 23.9 & 28.1 & 21.3 & 26.6 \\
\hline $\mathrm{KCF}$ & 121.3 & & $\sqrt{ }$ & 20.2 & 12.5 & 20.9 & 14.5 & 13.5 & 18.3 & 14.6 & 22.2 & 22.3 & 23.8 & 27.9 & 21.0 & 26.5 \\
\hline ACRST & 4.4 & $\sqrt{ }$ & $\sqrt{ }$ & 45.2 & 34.4 & 51.8 & 41.7 & 28.7 & 43.8 & 32.2 & 46.5 & 44.8 & 47.9 & 51.1 & 48.2 & 51.2 \\
\hline
\end{tabular}

Table 4: Ablation study of ACRST.

\begin{tabular}{lllllll}
\hline Method & RT & RS & ACL & DF & DS & SS \\
\hline ACRST_basic & $\times$ & $\times$ & $\times$ & $\times$ & 0.627 & 0.456 \\
ACRST_RT & $\sqrt{ }$ & $\times$ & $\times$ & $\times$ & 0.636 & 0.464 \\
ACRST_RT\&RS & $\sqrt{ }$ & $\sqrt{ }$ & $\times$ & $\times$ & 0.647 & 0.468 \\
ACRST_RT\&RS\&ACL & $\sqrt{ }$ & $\sqrt{ }$ & $\sqrt{ }$ & $\times$ & 0.653 & 0.474 \\
ACRST_full & $\sqrt{ }$ & $\sqrt{ }$ & $\sqrt{ }$ & $\sqrt{ }$ & 0.716 & 0.512 \\
\hline
\end{tabular}
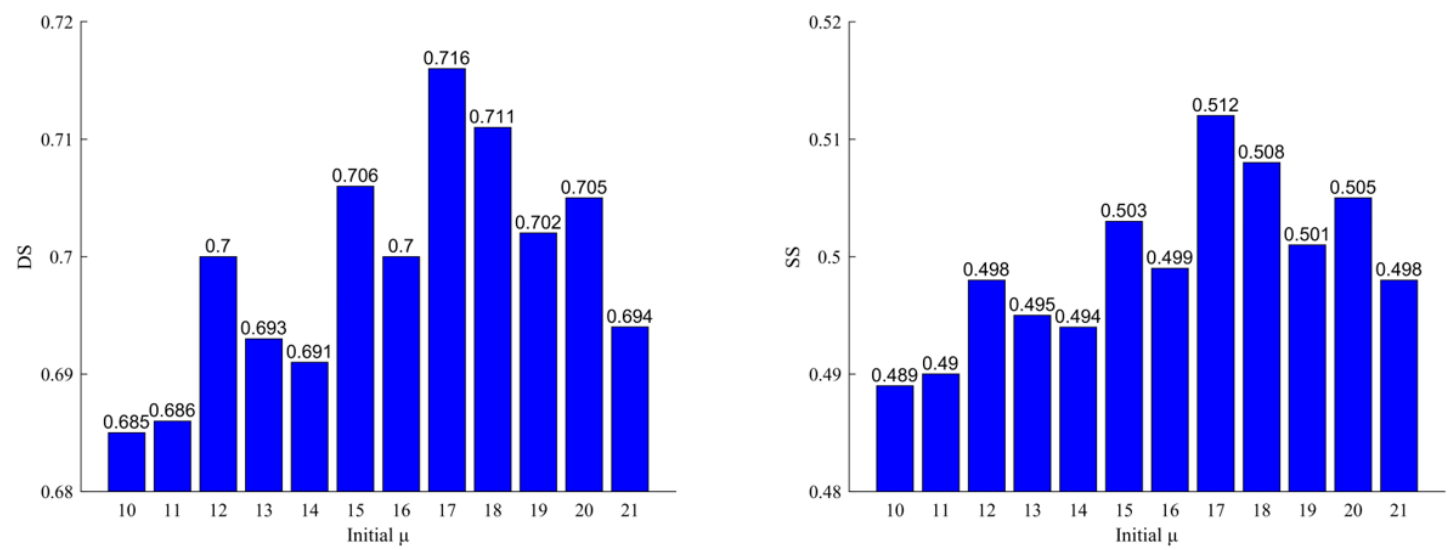

Figure 5: Effect of different values of $\mu_{\text {initial }}$ on the tracking performance. 
that our tracker performs favorably especially in some challenging scenes.

\section{REFERENCES}

[1] Hui Cheng, Lishan Lin, Zhuogi Zheng, Yuwei Guan, and Zhongchang Liu. 2017. An autonomous visionbased target tracking system for rotorcraft unmanned aerial vehicles. In 2017 IEEE/RSJ International Conference on Intelligent Robots and Systems (IROS). IEEE, 1732-1738.

[2] Jan Prokaj and G'erard Medioni. 2014. Persistent tracking for wide area aerial surveillance. In Proceedings of the IEEE Conference on Computer Vision and Pattern Recognition. 1186-1193.

[3] Teodor Tomic, Korbinian Schmid, Philipp Lutz, Andreas Domel, Michael Kassecker, Elmar Mair, Iris Lynne Grixa, Felix Ruess, Michael Suppa, and Darius Burschka. 2012. Toward a fully autonomous UAV: Research platform for indoor and outdoor urban search and rescue. IEEE robotics \& automation magazine 19 , 3 (2012), 46-56.

[4] Martin Danelljan, Gustav Hager, Fahad Shahbaz Khan, and Michael Felsberg 2015. Learning spatially regularized correlation filters for visual tracking. In Proceedings of the IEEE international conference on computer vision. 4310-4318.

[5] Matthias Mueller, Neil Smith, and Bernard Ghanem. 2017. Context-aware correlation filter tracking. In Proceedings of the IEEE Conference on Computer Vision and Pattern Recognition. 1396-1404.

[6] Feng Li, Cheng Tian, Wangmeng Zuo, Lei Zhang, and Ming-Hsuan Yang. 2018 Learning spatial-temporal regularized correlation filters for visual tracking. In Proceedings of the IEEE Conference on Computer Vision and Pattern Recognition. 4904-4913.

[7] David S Bolme, J Ross Beveridge, Bruce A Draper, and Yui Man Lui. 2010. Visual object tracking using adaptive correlation filters. In 2010 IEEE computer society conference on computer vision and pattern recognition. IEEE, 2544-2550.

[8] Joao F Henriques, Rui Caseiro, Pedro Martins, and Jorge Batista. 2012. Exploit ing the circulant structure of tracking-by-detection with kernels. In European conference on computer vision. Springer, 702-715.

[9] J. F. Henriques, R. Caseiro, P. Martins, and J. Batista. 2015. High-Speed Tracking with Kernelized Correlation Filters. IEEE Transactions on Pattern Analysis and Machine Intelligence 37, 3 (2015), 583-596.

[10] Martin Danelljan, Fahad Shahbaz Khan, Michael Felsberg, and Joost Van de Weijer 2014. Adaptive color attributes for real-time visual tracking. In Proceedings of the IEEE Conference on Computer Vision and Pattern Recognition. 1090-1097.

[11] Martin Danelljan, Gustav H“"ager, Fahad Shahbaz Khan, and Michael Felsberg. 2016. Discriminative scale space tracking. IEEE transactions on pattern analysis and machine intelligence 39, 8 (2016), 1561-1575.

[12] Yang Li and Jianke Zhu. 2014. A scale adaptive kernel correlation filter tracker with feature integration. In European conference on computer vision. Springer 254-265.

[13] Si Liu, Tianzhu Zhang, Xiaochun Cao, and Changsheng Xu. 2016. Structural correlation filter for robust visual tracking. In Proceedings of the IEEE Conference on Computer Vision and Pattern Recognition. 4312-4320.

[14] Ting Liu, Gang Wang, and Qingxiong Yang. 2015. Real-time part-based visual tracking via adaptive correlation filters. In Proceedings of the IEEE Conference on Computer Vision and Pattern Recognition. 4902-4912.

[15] Hamed Kiani Galoogahi, Ashton Fagg, and Simon Lucey. 2017. Learning background-aware correlation filters for visual tracking. In Proceedings of the IEEE international conference on computer vision. 1135-1143.

[16] Martin Danelljan, Goutam Bhat, Fahad Shahbaz Khan, and Michael Felsberg. 2017. Eco: Efficient convolution operators for tracking. In Proceedings of the
IEEE conference on computer vision and pattern recognition. 6638-6646.

[17] Martin Danelljan, Gustav Hager, Fahad Shahbaz Khan, and Michael Felsberg. 2016. Adaptive decontamination of the training set: A unified formulation for discriminative visual tracking. In Proceedings of the IEEE Conference on Computer Vision and Pattern Recognition. 1430-1438.

[18] Feng Li, Cheng Tian, Wangmeng Zuo, Lei Zhang, and Ming-Hsuan Yang. 2018. Learning spatial-temporal regularized correlation filters for visual tracking. In Proceedings of the IEEE Conference on Computer Vision and Pattern Recognition. 4904-4913.

[19] Ziyuan Huang, Changhong Fu, Yiming Li, Fuling Lin, and Peng Lu. 2019. Learning aberrance repressed correlation filters for real-time uav tracking. In Proceedings of the IEEE International Conference on Computer Vision. 2891-2900.

[20] Mengmeng Wang, Yong Liu, and Zeyi Huang. 2017. Large margin object tracking with circulant feature maps. In Proceedings of the IEEE Conference on Computer Vision and Pattern Recognition. 4021-4029.

[21] Jongwon Choi, Hyung Jin Chang, Sangdoo Yun, Tobias Fischer, Yiannis Demiris, and Jin Young Choi. 2017. Attentional correlation filter network for adaptive visual tracking. In Proceedings of the IEEE conference on computer vision and pattern recognition. 4807-4816.

[22] Chao Ma, Jia-Bin Huang, Xiaokang Yang, and Ming-Hsuan Yang. 2015. Hierarchical convolutional features for visual tracking. In Proceedings of the IEEE international conference on computer vision. 3074-3082.

[23] Ning Wang, Wengang Zhou, Oi Tian, Richang Hong, Meng Wang, and Houqiang Li. 2018. Multi-cue correlation filters for robust visual tracking. In Proceedings of the IEEE Conference on Computer Vision and Pattern Recognition. 4844-4853.

[24] Luca Bertinetto, Jack Valmadre, Joao F Henriques, Andrea Vedaldi, and Philip HS Torr. 2016. Fully convolutional siamese networks for object tracking. In European conference on computer vision. Springer, 850-865.

[25] Jack Valmadre, Luca Bertinetto, Joao Henriques, Andrea Vedaldi, and Philip HS Torr. 2017. End-to-end representation learning for correlation filter based tracking. In Proceedings of the IEEE Conference on Computer Vision and Pattern Recognition. 2805-2813.

[26] Matthias Mueller, Neil Smith, and Bernard Ghanem. 2016. A benchmark and simulator for uav tracking. In European conference on computer vision. Springer, 445-461.

[27] Chen Wang, Le Zhang, Lihua Xie, and Junsong Yuan. 2018. Kernel cross-correlator. In Thirty-Second AAAI Conference on Artificial Intelligence.

[28] Luca Bertinetto, Jack Valmadre, Stuart Golodetz, Ondrej Miksik, and Philip HS Torr. 2016. Staple: Complementary learners for real-time tracking. In Proceedings of the IEEE conference on computer vision and pattern recognition. 1401-1409.

[29] Kenan Dai, Dong Wang, Huchuan Lu, Chong Sun, and Jianhua Li. 2019. Visual Tracking via Adaptive Spatially-Regularized Correlation Filters. In The IEEE Conference on Computer Vision and Pattern Recognition (CVPR).

[30] Ning Wang, Yibing Song, Chao Ma, Wengang Zhou, Wei Liu, and Houqiang Li. 2020. Unsupervised Deep Tracking. In 2019 IEEE/CVF Conference on Computer Vision and Pattern Recognition (CVPR).

[31] Yi Wu, Jongwoo Lim, and Ming-Hsuan Yang. 2013. Online object tracking: A benchmark. In Proceedings of the IEEE conference on computer vision and pattern recognition. 2411-2418.

[32] Andrea Vedaldi and Karel Lenc. 2015. Matconvnet: Convolutional neural networks for matlab. In Proceedings of the 23rd ACM international conference on Multimedia. 689-692.

[33] Stephen Boyd, Neal Parikh, and Eric Chu. 2011. Distributed optimization and statistical learning via the alternating direction method of multipliers. Now Publishers Inc. 